\title{
Rezension „Open Source Software“
}

\author{
Matthias Knoll (D)
}

Angenommen: 7. Dezember 2020 / Online publiziert: 16. Dezember 2020

(C) Der/die Autor(en) 2020

Till Jaeger und Axel Metzger Open Source Software - Rechtliche Rahmenbedin-
gungen der Freien Software
ISBN 978-3-406-73497-7, C.H. Beck oHG, 5. Aufl., München 2020, 394 S., $89 €$

All jene, die sich für den Einsatz quelloffener Software begeistern, ob als Anwender (auch im kommerziellen oder behördlichen Umfeld) oder Entwickler, wissen nur zu gut um häufig diffus begründete ,rechtliche Probleme“ in Diskussionen des Für und Wider konkreter Entwicklungs-, Beschaffungs- und Einsatzszenarien. Da ist Expertenrat gefragt.

Die beiden Autoren veröffentlichten vor 21 Jahren in der GRUR International den ersten gemeinsamen Fachbeitrag zu Open Source Software und dem deutschen Urheberrecht und damit den ersten deutschsprachigen Beitrag dieses damals noch relativ neuen Entwicklungsmodells Open Source überhaupt. Nun arbeiten sie mit dem vorliegenden Buch bereits seit 18 Jahren in bewährter Weise den aktuellen Stand des Rechts in diesem Themengebiet auf. Beide Autoren können also mit Fug und Recht als Instanzen auf diesem Gebiet angesehen werden.

Kam die erste Auflage noch mit knapp 230 Seiten aus, erkennt man bezogen auf die aktuelle Ausgabe in der Verdopplung der Seitenzahl, dass das Rechtsgebiet seither nicht nur an Bedeutung, sondern auch an Komplexität deutlich zugenommen hat.

Und tatsächlich: Viele wichtige und häufig eingesetzte Anwendungssysteme bestehen ganz oder in Teilen aus Open Source, auf zahlreichen Servern, gerade in der Cloud, laufen Linux-Derivate und auch sehr viele „Embedded Systems“ wie Router,



Hochschule Darmstadt, Darmstadt, Deutschland

E-Mail: matthias.knoll@h-da.de 
Telefone oder Smart-TVs setzen ein modifiziertes Linux und anderen quelloffenen Code ein.

Entsprechend genau muss hinsehen, wer sich beim Einsatz nicht im Dschungel der Lizenzformen und anderer juristischer Feinheiten verirren möchte. Orientierung ist deshalb gefragt. Zur Herstellung einer solchen Orientierung ist gute Strukturierung und fundierte Aufklärung zwingend notwendig, nur so können die vielfältigen Vorteile von Open Source Software zielführend genutzt werden.

Genau diese Orientierung ermöglicht dieses sehr umfassende Buch zum Thema in bewährter Weise.

Nach einer Einführung zu Begriff, Entstehung, Entwicklung und der wirtschaftlichen Bedeutung von Freier Software werden in sechs weiteren Kapiteln alle Aspekte des Themenkomplexes strukturiert beleuchtet. Gleich zu Beginn, in Abschnitt A II des ersten Kapitels, wird die wichtige Diskussion dargestellt, die vom ursprünglichen Begriff ,free software“ zum heute gebräuchlichen Begriff „open source software“ führte. Im weiteren Verlauf werden die Beziehung der öffentlichen Hand zu Freier Software ebenso thematisiert wie Embedded Systems/IoT, der wichtige Dienstleistungsmarkt und SaaS-/Cloud-Lösungen in diesem Umfeld. Überaus interessant sind die Darstellungen zu Compliance-Fragen, die unter anderem mit Blick auf Haftungsfragen verdeutlichen, dass beim Einsatz von Open Source Software (auch im Rahmen des Bezugs von Produkten und Software von Drittanbietern) gleiche Compliance-Regeln angewandt werden müssen, wie überall sonst im Unternehmen, wenngleich mit der Ausgestaltung eines Open-Source-Compliance-Systems durchaus noch anspruchsvolle Fragen verbunden sind. Positiv ist, dass die Autoren hier zahlreiche Hinweise geben, wie vorgegangen werden könnte, um zumindest die wichtigsten Fragen im jeweiligen Kontext besser beantworten zu können.

Mit den folgenden vier Kapiteln nehmen mit insgesamt etwa 250 Seiten die Themen Lizenzfragen, Urheberrecht, Vertragsrecht, Haftung und Gewährleistung sowie die gewerblichen Schutzrechte den größten Raum ein. Das Buch schließt mit einem Blick auf das Wettbewerbsrecht und das internationale Privatrecht sowie einem Vergleich mit dem Recht in wichtigen Ländern, beispielsweise den USA, Großbritannien oder Frankreich. Dieser Abschnitt ist wegen der Internationalität der Open-Source-Community für Entwicklung und Vertrieb besonders interessant, bedeutsam und notwendig. Denn häufig findet ausländisches Recht Anwendung. Entsprechend widmet sich dieser Abschnitt auf etwa 13 Seiten den wichtigsten Fragen.

Ein sehr informativer Anhang zur Rechtsprechung, zur seit 2007 unverändert gültigen, in 10 Punkten niedergelegten ,The Open Source Definition“ der Open Source Initiative (https://opensource.org/osd) und den wichtigsten Lizenztexten rundet diese Auflage ab.

Es fällt sehr schwer, das geballte, gut strukturiert dargestellte Wissen, das nur sehr wenige Detailfragen unbeantwortet lässt, mit wenigen Sätzen zusammenfassend darzustellen. Was sich hingegen leicht sagen lässt, ist, dass das über die Jahre gewachsene Buch einen auch für Nichtjuristen sehr guten, leicht lesbaren Über- und Einblick in alle Bereiche dieses Themas gibt. Es verdeutlicht die Vielzahl an Fallstricken und komplexen Konstellationen, ermutigt jedoch durch die Art der Darstellung und Vermittlung, sich trotz mancher Schwierigkeit mit der Entwicklung und/oder 
dem Einsatz von Open Source Software zu befassen und die sich daraus ergebenden Chancen unter sorgfältiger Abwägung der Risiken zu nutzen. Damit leistet es einen umfassenden und überaus wichtigen Beitrag zur Verbreitung von Open Source Software und zur Klarstellung mancher alter Missverständnisse und Irrtümer. Es bleibt zu hoffen, dass das Autorenteam auch weiterhin am Puls der rechtlichen Entwicklungen bleiben kann, um in entsprechenden Abständen eine Aktualisierung vorzulegen, die nicht nur für Fachleute lesenswert ist.

Funding Open Access funding enabled and organized by Projekt DEAL.

Open Access Dieser Artikel wird unter der Creative Commons Namensnennung 4.0 International Lizenz veröffentlicht, welche die Nutzung, Vervielfältigung, Bearbeitung, Verbreitung und Wiedergabe in jeglichem Medium und Format erlaubt, sofern Sie den/die ursprünglichen Autor(en) und die Quelle ordnungsgemäß nennen, einen Link zur Creative Commons Lizenz beifügen und angeben, ob Änderungen vorgenommen wurden.

Die in diesem Artikel enthaltenen Bilder und sonstiges Drittmaterial unterliegen ebenfalls der genannten Creative Commons Lizenz, sofern sich aus der Abbildungslegende nichts anderes ergibt. Sofern das betreffende Material nicht unter der genannten Creative Commons Lizenz steht und die betreffende Handlung nicht nach gesetzlichen Vorschriften erlaubt ist, ist für die oben aufgeführten Weiterverwendungen des Materials die Einwilligung des jeweiligen Rechteinhabers einzuholen.

Weitere Details zur Lizenz entnehmen Sie bitte der Lizenzinformation auf http://creativecommons.org/ licenses/by/4.0/deed.de. 